\title{
Efeito do gel da babosa (Aloe barbadensis Mill.) associado ao ultrassom em processo inflamatório agudo
}

\author{
MAIA-FILHO, A.L.M. ${ }^{1 *}$; SILVA,V.S. ${ }^{1}$; BARROS,T.L. ${ }^{1}$; COSTA, C.L.S. ${ }^{1}$ MAIA, E.P.V.D. ${ }^{1}$; ARAUUJ, K.S. ${ }^{1}$; SANTOS, \\ I.M.S.P. ${ }^{1}$; VILLAVERDE, A.G.J.B. ${ }^{2}$; CARVALHO, F.A.S. ${ }^{2}$; CARVALHO, R.A. ${ }^{2}$ \\ ${ }^{1}$ Faculdade Integral Diferencial - FACID, Avenida Rio Poty, 2381, Horto Florestal, CEP: 64049-410, Teresina-Brasil

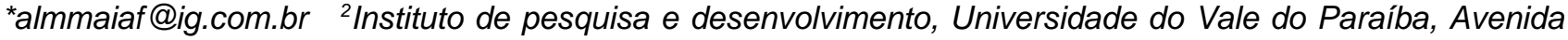 \\ Shishima Hifumi, 2911, Urbanova, CEP: 12244-000, São José dos Campos-Brasil
}

RESUMO: O presente trabalho teve por objetivo analisar a ação antiinflamatória do gel da Babosa a $2 \%$ (Aloe barbadensis Mill.) associado ao Ultrassom pulsátil no modelo de edema de pata. Foram utilizados 25 ratos Wistar, $(200-250 \mathrm{~g})$, divididos em 5 grupos de 5 animais cada. Grupo1 (controle): ratos tratados com solução salina a 0,9\%; Grupo 2: ratos tratados topicamente com gel de $A$. barbadensis Mill. a 2\%; Grupo 3: animais tratados com Ultrassom; Grupo 4: ratos tratados com gel de $A$. barbadensis Mill. a 2\% associado ao Ultrassom; Grupo 5 (controle positivo): ratos tratados com Indometacina na dose de $5 \mathrm{mg} \mathrm{Kg}^{-1}$. Os animais dos grupos 1 e 5 receberam os respectivos tratamentos por via intra-peritoneal 30 minutos antes da injeção intraplantar de carragenina e os grupos 2, 3 e 4 foram tratados por aplicação tópica de gel de $A$. barbadensis Mill. a $\%$, Ultrassom pulsátil e gel de A. barbadensis Mill. associado ao Ultrassom respectivamente 15 minutos após a indução do edema. Os animais do grupo 04 demonstraram redução significativa do edema quando comparados ao grupo controle, ao mesmo tempo, que se mostrou comparável à indometacina. Observou-se que o gel de aloe associado à fonoforose é capaz reduzir a formação do edema de pata em ratos.

Palavras-chaves: Aloe barbadensis, ultrassom, inflamação, fonoforese

\begin{abstract}
Effect of aloe (Aloe barbadensis Mill.) gel combined with ultrasound in the acute inflammatory process. This work aimed to evaluate the anti-inflammatory action of $2 \%$ aloe (Aloe barbadensis Mill.) gel combined with pulsed ultrasound in the paw edema model. Twenty-five Wistar rats (200-250 g) were divided into 5 groups of 5 animals each. Group1 (control): rats treated with $0.9 \%$ saline; Group 2: rats topically treated with $2 \%$ aloe gel; Group 3: rats treated with ultrasound; Group 4: rats treated with $2 \%$ aloe gel combined with ultrasound; Group 5 (positive control): rats treated with indomethacin at $5 \mathrm{mg} \mathrm{Kg}^{-1}$. Animals of groups 1 and 5 were intraperitoneally treated 30 min before intraplantar carrageenan injection and groups 2, 3 and 4 were treated by topical application of $2 \%$ aloe gel, pulsed ultrasound and aloe gel combined with ultrasound, respectively, $15 \mathrm{~min}$ after edema induction. Animals of group $4 \mathrm{had}$ a significant reduction in edema relative to controls and showed to be comparable to indomethacin. Aloe gel combined with phonophoresis is capable of reducing paw edema formation in rats.
\end{abstract}

Key words: Aloe barbadensis, ultrasound, inflammation, phonophoresis

\section{INTRODUÇÃO}

Após muitas controvérsias, o uso de plantas medicinais começa a ter apoio científico em todos os recantos do planeta. O Brasil e outros países, com apoio da Organização Mundial de Saúde, estão começando a resgatar a medicina popular, em que as plantas medicinais ressurgiram com força e vigor.
Muitas ervas têm uso milenar, posto que há citação de várias em papiros do antigo Egito. Atualmente, encontra-se facilmente em jornais, revistas e também em publicações da área da saúde uma variada gama de artigos sobre plantas medicinais, que estão sendo utilizadas, cada vez mais, com respaldo científico.

Recebido para publicação em 16/03/2009

Aceito para publicação em 11/12/2010

Rev. Bras. PI. Med., Botucatu, v.13, n.2, p.146-150, 2011. 
Entre as ervas mais difundidas, encontra-se a californiana Aloe barbadensis. A espécie $A$. barbadensis (babosa) é uma planta tropical da família Liliaceae que cresce muito facilmente em climas quentes e secos. Muitos produtos medicinais e cosméticos são produzidos a partir da mucilagem encontrada no centro das folhas da aloe (Maenthaisong et al., 2007). A planta é frequentemente utilizada no tratamento de queimaduras, ulcerações da pele e ferimentos estão bem documentados e, provavelmente, estes são os efeitos mais conhecidos (Franco, 2006).

A inflamação é resposta orgânica local ou sistêmica, de magnitude variável, desencadeada por diversos fatores, tendo como fim proteger o organismo contra agressões. Esta resposta ocorre através de processo de regulação que mantém o equilíbrio das diversas funções e composições químicas do corpo, ou seja, a reação inflamatória é o mecanismo fisiopatológico básico em resposta a diversas doenças, sendo representada por um conjunto de reações locais e gerais do organismo (Contran et al., 2005). A presença de mediadores químicos no processo inflamatório faz com que a inflamação mantenha características uniformes mesmo sendo produzida por diferentes irritantes sendo que o aumento da permeabilidade vascular pode ser originado de mecanismos diretos, em que o próprio agente agressor atua sobre a parede vascular ou indiretos em que há ação de mediadores químicos (Rang et al., 2007).

As doenças de origem inflamatória e não infecciosas são, tradicionalmente, tratadas com antiinflamatórios esteroidais (AIEs) ou não-esteroidais (AINEs) (Rang et al., 2007). Entretanto, terapias físicas como estimulação elétrica (Gersh, 1990), ondas curtas (Ko et al., 2001), irradiação infravermelha, acupultura e ultrassom terapêutico de baixa e média intensidade (Ziskin et al., 1990) e laser de baixa potência vêm ganhando espaço como alternativa de tratamento antiinflamatório. Muitos trabalhos vem sendo desenvolvidos nesta área relatando vantagens em relação à terapia medicamentosa devido aos menores efeitos adversos produzidos por terapias físicas.

As oscilações cinéticas ou mecânicas do Ultrassom (US), produzidas por transdutor vibratório, podem ser aplicadas sobre a pele com finalidade terapêutica, atravessando-a e penetrando no organismo em diferentes profundidades. Devido a freqüência ( 1 - 3 e $5 \mathrm{MHz}$ ), saem do campo de percepção do ouvido humano (Machet, \& Boucaud, 2002).

Entre os efeitos biológicos promovidos pelo US estão a modulação da microcirculação e permeabilidade vascular; efeito analgésico e espamolítico; incremento da flexibilidade dos tecidos ricos em colágeno, com redução da rigidez articular e contratura e aumento do metabolismo local, com estimulação das funções celulares para a regeneração celular (Polacow et al., 2005).

A estimulação ultra-sônica, pulsada em ratos submetidos à Incisão cirúrgica na cavidade abdominal (celiotomia), mostrou maior proporção volumétrica de células polimorfonucleares (PMN) e fibroblastos quando comparados com animais que receberam aplicação de US no modo contínuo, demonstrando que a aplicação de US no modo pulsado auxilia na cicatrização (Olsson et al., 2006). A estimulação com ultrassom, especialmente no modo pulsado com intensidade baixa, induz aceleração do reparo tecidual devido o aumento da angiogênese, do tecido de granulação, do número de fibroblastos e da síntese de colágeno, e a diminuição de leucócitos e macrófagos na área lesada (Mendonça et al., 2006; Borges, 2006).

Apesar da grande divergência de parâmetros e não elucidação do mecanismo de ação parece haver consenso que o ultrassom pode exercer a ação próinflamatória através do incremento da síntese de colágeno e fibroblastos e promovendo a liberação de histamina, macrófagos e monócitos (Guirro, 2004).

As ondas ultra-sônicas são absorvidas pelos tecidos e transformadas em calor, ocorrendo principalmente em nível molecular, sendo as proteínas os tecidos que mais absorvem. Ao penetrarem nos tecidos, provocam vibração celular (micromassagem), produzindo o aumento da permeabilidade da membrana, acelerando assim, a velocidade de difusão iônica através dela (Rosa Filho et al., 2003). O ultrassom tem potencial de acelerar o curso normal da inflamação desde que o estímulo inflamatório seja removido. Essa aceleração pode dever-se à suave agitação do líquido dos tecidos que pode aumentar a taxa de fagocitose e o movimento das partículas e células. Deve-se observar que o ultrassom tem ação pró-inflamatória e não antiinflamatória. Às vezes considera-se que o ultrassom terapêutico resulta em aumento no fluxo sanguíneo local (Parizoto et al., 2003).

A fonoforese é definida como a migração de moléculas de drogas através da pele sob a influência do ultrassom. Teoricamente, a fonoforese é possível utilizando as forças de correntes acústicas que existem no campo de ultrassom (Mitragotri, 2000). Contudo é questionável se essas forças são fortes o suficiente para produzir movimento resultante para a frente capaz de empurrar todas as drogas através da pele até o tecido alvo. Além disso, é geralmente difícil determinar se o efeito biológico de droga aplicada topicamente é resultado da ação direta no tecido alvo subjacente ou devido a efeito sistêmico (Kitchen, 2003). O ultrassom aumenta a penetração transcutânea por pressão somática, podendo atingir,

Rev. Bras. PI. Med., Botucatu, v.13, n.2, p.146-150, 2011. 
em média, entre 4-5 cm de profundidade (Machet \& Boucaud, 2002).

Desta forma, o presente trabalho teve por intenção avaliar a associação do ultrassom com a Aloe barbadensis Mill. (babosa) para o tratamento da inflamação utilizando modelo de tendinites crônicas em ratos.

\section{MATERIAL E MÉTODO}

Os animais foram provenientes do biotério da Faculdade Integral Diferencial-FACID. Mantidos a temperatura constante, obedecendo a ciclo de claro/ escuro de $12 \mathrm{~h}$ e com acesso livre a comida e água. O protocolo experimental deste trabalho foi aprovado pelo Comitê de Ética em Pesquisa da FACID sob número de protocolo 097/2007

Foram utilizados 25 animais da espécie Rattus norvegicus variedade Wistar (200-250 g), divididos em cinco grupos de cinco animais, de acordo com o tratamento, e foram demarcadas com caneta de tinta vermelha nas caudas para que não houvesse risco de repetição de cobaias. 0,9\%;

Grupo 1: ratos tratadas com solução salina a

Grupo 2: ratos tratadas com gel de Aloe barbadensis. a 2\% com aplicação tópica;

Grupo 3: ratos tratadas com ultrassom de 3 $\mathrm{MHz}$ no modo pulsátil com gel a base de água durante 4 minutos cada;

Grupo 4: ratos tratadas com gel de Aloe barbadensis a $2 \%$ associado ao ultrassom de $3 \mathrm{MHz}$ no modo pulsátil durante 4 minutos cada;

Grupo 5: ratos tratadas com Indometacina na dose de $5 \mathrm{mg} \mathrm{Kg}^{-1}$.

O processo de indução de edema se deu através da injeção intra-plantar de carragenina (Winter et al.,1962; Franco et al., 2005). Os animais dos grupos 1 e 5 foram tratados por injeção intra-peritoneal solução salina e indometacina 30 minutos antes da injeção intra-plantar de $0,1 \mathrm{~mL}$ de carragenina $0,5 \%$ em uma das patas e os grupos 2, 3 e 4 foram tratados 15 minutos após a injeção de carragenina por aplicação tópica de gel de $A$. barbadensis a $2 \%$, ultrassom pulsátil com gel a base de água e gel de A. barbadensis associados ao ultrassom pulsátil respectivamente.

O gel $A$. barbadensis a $2 \%$ foi adquirido em farmácia de manipulação na cidade de Teresina-PI. Os tratamentos tópicos eram realizados 15 minutos após cada leitura feita através do hidropletismômetro. As leituras foram realizadas nos tempos de 30,60, 120,240 e 480 minutos após a injeção de carragenina (Franco et al., 2005).

Todos os tratamentos e leituras foram realizados na pata traseira direita de cada animal. Para a intervenção os animais do grupo 3 e 4 foram colocados em câmara anestésica, utilizando o éter como anestésico volátil. E após o término da pesquisa os animais foram sacrificados por indução excessiva da anestesia.

Após a coleta de dados foi realizado a analise estatística utilizando o programa estatístico Prisma 3.0 pelo método one way ANOVA (não paramétrico) seguido do Teste de Tukey, com intervalo de confiança em 95\% (Kupeli et al., 2007).

\section{RESULTADO E DISCUSSÃO}

Fatores quimiotáticos e vasoativos como a norepinefrina e serotonina são secretados imediatamente após a lesão, promovendo vasodilatação nos primeiros 5 a 10 minutos modificando o tônus vascular por meio da vasodilatação, contribuindo para o aumento da permeabilidade vascular e, consequente, o aumento do número de células inflamatórias (monócitos e neutrófilos) para o sítio da lesão (Willoughby et al., 2000).

Conforme os dados apresentados na Figura 1, que representa o registro da variação do volume da pata do animal da espécie Rattus norvegicus em edema induzidos por carragenina, tratados com solução salina e gel de Aloe barbadensis a $2 \%$.

O gel de Aloe barbadensis contém a mucilagem característica das folhas da espécie e cuja constituição se sobressaem os polissacarídeos. A mucilagem possui entre $80-94 \%$ de carboidratos, principalmente de glicose $(22-35 \%)$, galactose (11$15 \%)$, manose $(6.0-6.4 \%)$, ramnose $(1.6-4.0 \%)$, arabinose (2.8-3.2\%) e xilose (1.8-2.0\%) (Cui et al., 2006), e devido a alta hidrofilidade, estas substâncias não atravessam as barreiras impostas pela pele ou atravessam com muita dificuldade (Simões et al., 2004). Observou-se que o uso tópico do gel de aloe sem associação e do ultrassom sozinho não demonstraram redução significativa do edema dos respectivos grupos, em relação ao controle negativo.

Constatou-se ainda a inexistência de atividade comparativamente significativa entre o uso tópico do gel com Aloe barbadensis. $2 \%$ e o uso do ultrassom com gel a base de água (Figura 1). Não foi possível a visualização do efeito antiinflamatório atribuído ao ultrassom durante a fase aguda do processo inflamatório através de efeitos não térmicos conforme descrito na literatura (Low \& Reed, 2002).

$\mathrm{O}$ gel de Aloe barbadensis associado ao ultrassom pulsado apresentou redução significativa do edema de pata em Rattus norvegicus quando confrontado com o grupo controle (Figura 2). $\mathrm{Na}$ comparação do grupo que recebeu o antiinflamatório não esteroidal indometacina com os animais tratados com fonoforese e gel de aloe não se percebe diferença estatisticamente significativa entre os dois grupos quanto à intensidade do edema. Observou-se que o

Rev. Bras. PI. Med., Botucatu, v.13, n.2, p.146-150, 2011. 
efeito do ultrassom é potencializado com a associação do gel de Aloe barbadensis, pois nota-se que o edema teve pico até 30 minutos, sendo seguido por declínio progressivo nos intervalos de mensuração de 60, 120, 240 e 480 minutos, mostrando significância estatística entre os tratamentos e efeito comparável à indometacina (Figura 2).

Aliado aos princípios ativos existentes na $A$. barbadensis, a fonoforese com os efeitos da energia ultrassônica abre caminhos para o transporte das substâncias, aumentando a efetividade do mesmo, sendo assim um dos recursos em que a fisioterapia encontra maiores condições de acelerar a difusão dos princípios ativos existesntes Aloe barbadensis proporcionando a redução do processo inflamatório (Kitchen, 2003).

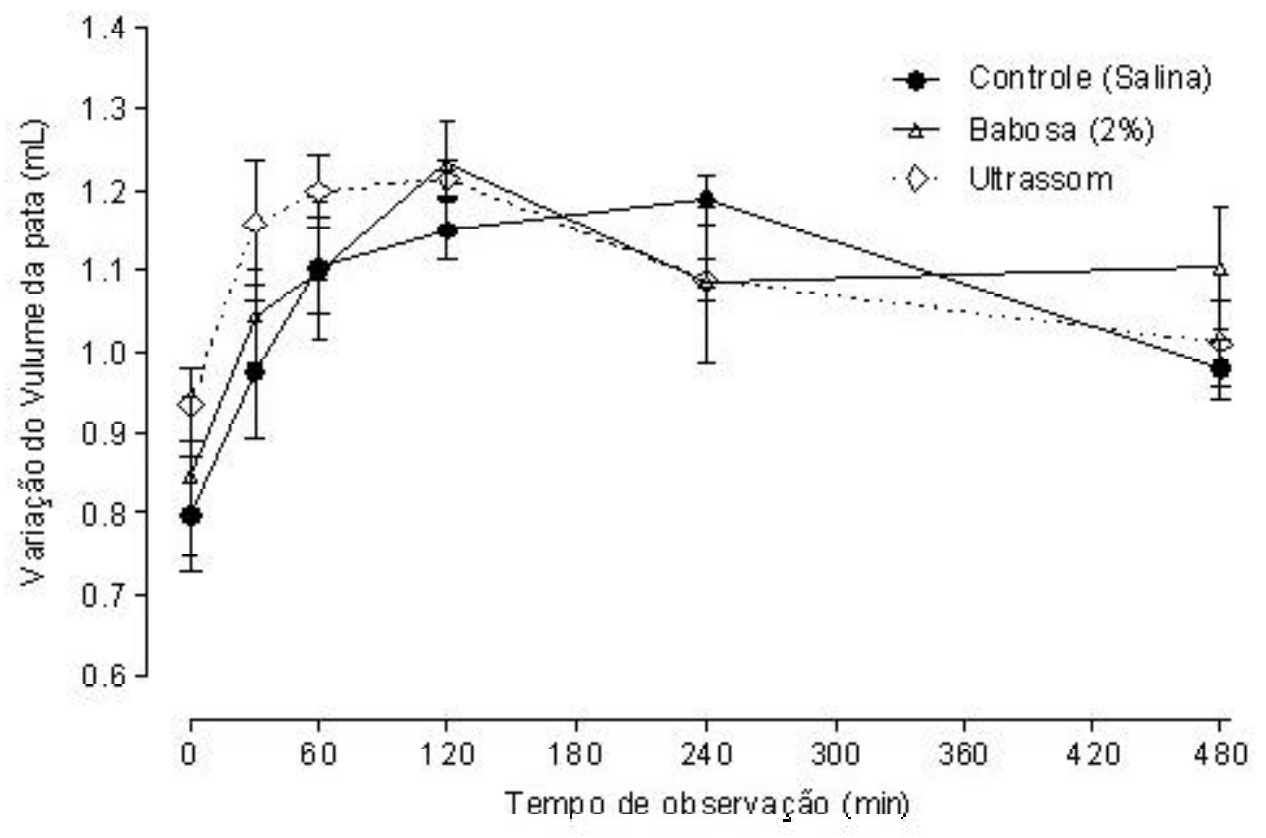

FIGURA 1. Registro da variação do volume da pata do animal da espécie Rattus norvegicus em edema induzidos por carragenina tratados com solução salina, gel de Aloe barbadensis $2 \%$ e ultrassom

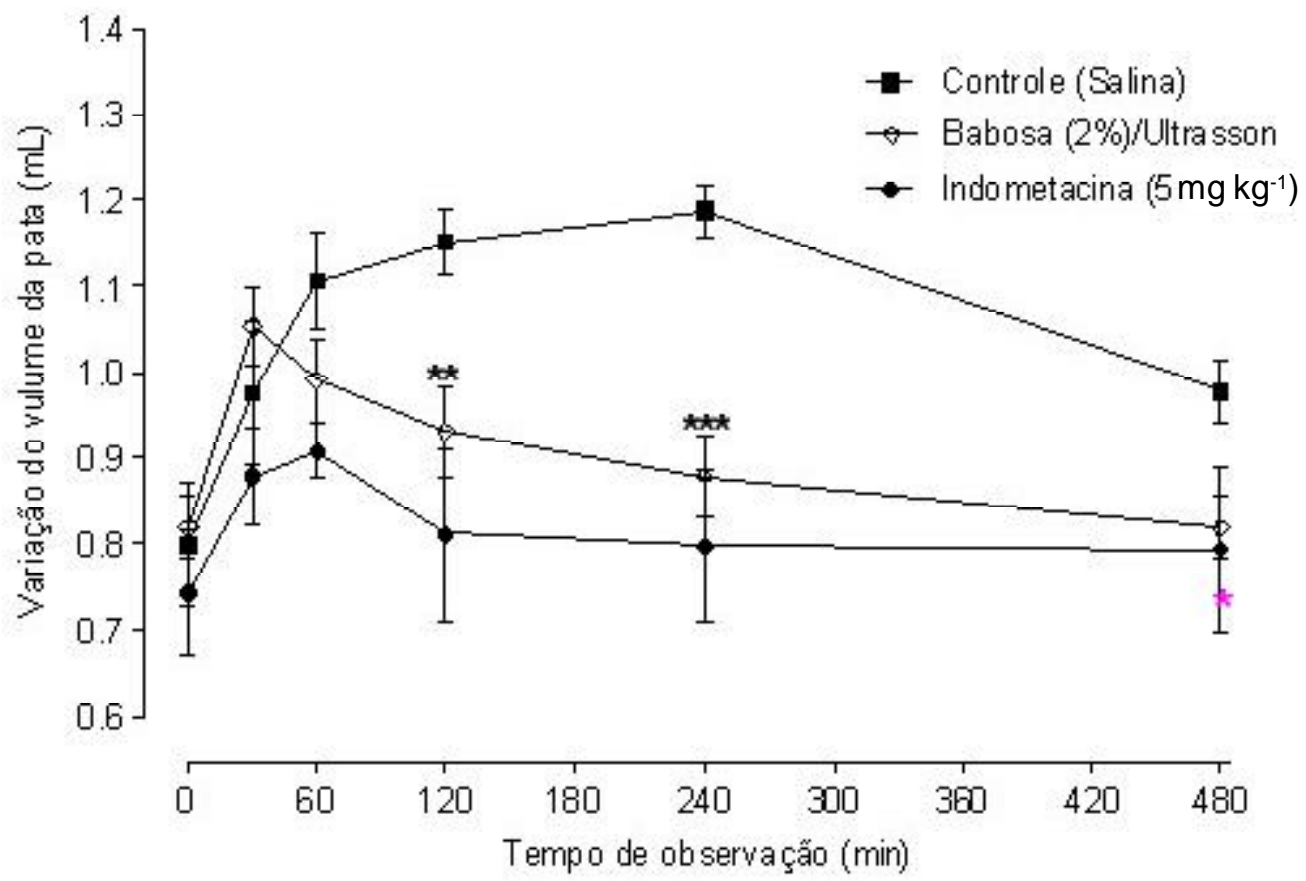

FIGURA 2. Registro da variação do volume da pata do animal da espécie Rattus norvegicus em edema induzidos por carragenina tratados com solução salina, indometacina e fonoforese associada ao gel de Aloe barbadensis $2 \%$. $\left.{ }^{* *} p<0,05 ;{ }^{* * *} p<0,01\right)$. 
O efeito observado com a associação advém da facilitação na penetração dos princípios ativos existentes na Aloe barbadensis pelo efeito do US proporcionando declínio progressivo e significativo do processo inflamatório agudo, vindo a corroborar as hipóteses iniciais.

Dada a complexidade da reação, o processo inflamatório é ainda tema de numerosas pesquisas no meio científico. Essa complexidade se dá pela interação simultânea no local inflamado de vários componentes orgânicos, locais e sistêmicos, tais como microcirculação sanguínea e linfática, células e fibras do tecido conjuntivo, sistema endócrino e sistema nervoso (Silva \& Garcia, 1972).

\section{CONCLUSÃO}

Após a realização dos ensaios e da observação dos resultados, pode-se concluir que a utilização do gel enriquecido a $2 \%$ com Aloe barbadensis Mill. apresentou marcante atividade antiinflamatória em edema de pata de ratos quando associado ao ultrassom pulsado, comparável àquela da indometacina.Estudos complementares são necessários para determinar a validade da utilização do gel de Aloe associado ao ultrassom como técnica auxiliar em fisioterapia.

\section{REFERÊNCIA}

BORGES, F. Dermato-funcional: modalidades terapêuticas nas disfunções estéticas. São Paulo: Phorte, 2006. 544p.

CONTRAN, R.S.; KUMAR, V.; ROBBINS, S.L. Fundamentos de Robbins: patologia estrutural e funcional. 7.ed. Rio de Janeiro: Guanabara Koogan, 2005.1485p.

CUI, S.W. et al. Synergisms between yellow mustard mucilage and galactomannans and applications in food products - A mini review. Advances in Colloid and Interface Science, v.128-30, n.1, p.249-56, 2006. Disponível em: <http://www.periodicos.capes.gov.br>. Acesso em: 15 dez. 2007.

FRANCO, A.D. et al. Análise do efeito do ultra-som no edema Inflamatório agudo-estudo experimental. Fisioterapia Movimento, v.8, n.2, p.19-24, 2005.

FRANCO, L.C.L. Uso de plantas medicinais tem apoio científico. 2006. Disponível em: <http://www.feridologo. com.br/fitoterapia2.htm>. Acesso: 15 dez. 2007.

GERSH, M.R. Electroterapy in rehabilitation. Philadelphia: F.A. Davis Co., 1990. p.149-96.

GUIRRO, E.C.O.; GUIRRO, R.R.J. Fisioterapia dermatofuncional: fundamentos, recursos e patologias. 3.ed. São Paulo: Manole, 2004. 560p.

KITCHEN, S. Eletroterapia prática baseada em evidências. 11.ed. São Paulo: Manole, 2003. 360p. KO, J.H.; CHEN, H.S.; CHE, N.L.M. Treatment of lateral epicondylitis of the elbow with shock waves. Clinical Orthopedic, v.1, n.387, p.60-7, 2001.

KUPELI, E. et al. Bioassay-guided isolation of antiinflammatory and antinociceptive glycoterpenoids from the flowers of Verbascum lasianthum Boiss. ex Bentham. Journal of Ethnopharmacology, v.110, n.3, p.444-50, 2007.

LOW, J.; REED. A. Therapeutic ultrasound. In: LOW, J.; REED. A. (Eds.). Electrotherapy explained: principles and practice. Philadelphia: Elsevier Health Science, 2002. 472p.

MACHET, L.; BOUCAUD, A. Phonophoresis: efficiency, mechanisms and skin tolerance. International Journal of Pharmaceutics, v.243, n.1, p.1-15, 2002.

MAENTHAISONG, R. et al. The efficacy of Aloe vera used for burn wound healing: A systematic review. Burns, v.33, n.6, p.713-8, 2007. Disponível em: <http://www. periodicos.capes.gov.br>. Acesso em: 09 mar. 2009. MENDONÇA, A.C. et al. Efeitos do ultra-som pulsado de baixa intensidade sobre a cicatrização por segunda intenção de lesões cutâneas totais em ratos. Acta Ortopédica Brasileira, v.14, n.3, p.152-7, 2006.

MITRAGOTRI, S. Synergistic effect of enhancers for transdermal drug delivery. Pharmaceutical Research, v.17, n.11, p.1354-9, 2000.

OLSSON, D.C. et al. Estimulação ultrasônicapulsada e contínua no processo cicatricial de ratos submetidos à celiotomia. Revista Ciência Rural, v.36, n.3, p.61-6, 2006. PARIZOTTO, N.A. et al. Utilização da Fonoforese em desordens músculo-esqueléticas: uma meta-análise. Revista Brasileira de Fisioteria, v.7, n.1, p 9-15, 2003. POLACOW, M.L.O. et al. Efeito do ultra-som e do dpantenol na regeneração tegumentar. Revista Brasileira de Fisioterapia, v.9, n.3, p.365-71, 2005.

RANG, H.P. et al. Farmacologia. 6.ed. São Paulo: Elservier, 2007. 920p.

ROSA FILHO, B.J. et al. Ultra-som. Fisioweb, 2003. Disponível em: <http://www.wgate.com.br/conteudo/ medicinaesaude/fisioterapia/eletro/ultra_som2.htm>. Acesso em: 15 dez. 2007

SILVA, M.R.; GARCIA, L.J. Chemical mediators of the acute inflammatory reaction. Oxford: Pergram, 1972. 425p.

SIMÕES, C.M.O. et al. Farmacognosia: da planta ao medicamento. 5.ed. Porto Alegre/Florianópolis: Editora da UFRGS/Editora da UFSC, 2004. 1102p.

WILLOUGHBY, D.A. et al. Resolution of inflammation. International Journal of Immunopharmacology, v.22, n.12, p.1131-5, 2000.

WINTER, C.A.; RISLEY, E.A.; NUSS, G.W. Carrageenaninduced o edemain the hind paw of rat as an assay for anti-inflammatory activity. Expert Opinion on Biological Therapy, v.111, p.544-7,1962.

ZISKIN, M.C.; MCDIAMID, T.; MICHLOVITZ, S.I. Therapeutic ultrasound. In: MICHLOVITZ, S.I. Thermal agents in rehabilitation. 2.ed. Philadelphia: F.A. Davis Co., 1990. p.134-69.

Rev. Bras. PI. Med., Botucatu, v.13, n.2, p.146-150, 2011. 